\title{
Dielectric properties of a bisimidazolium salt with dodecyl sulfate anion doped with carbon nanotubes
}

\author{
Doina Manaila Maximean ${ }^{* 1}$, Viorel Cîrcu ${ }^{2}$ and Constantin Paul Ganea ${ }^{* 3}$
}

\author{
Full Research Paper \\ Address: \\ ${ }^{1}$ University Politehnica of Bucharest, Department of Physics, 313 Spl. \\ Independentei, 060042, Bucharest, Romania, ${ }^{2}$ Department of \\ Inorganic Chemistry, University of Bucharest, 23 Dumbrava Rosie st, \\ sector 2, Bucharest 020464, Romania and ${ }^{3}$ National Institute of \\ Materials Physics, POBox MG 07, 077125 Magurele, Romania \\ Email: \\ Doina Manaila Maximean* - doina.manaila@physics.pub.ro; \\ Constantin Paul Ganea* - paul.ganea@infim.ro \\ * Corresponding author \\ Keywords: \\ activation energy; carbon nanotubes; dielectric spectroscopy; ionic \\ liquid crystal; relaxation time
}

Beilstein J. Nanotechnol. 2018, 9, 164-174.

doi:10.3762/bjnano.9.19

Received: 22 August 2017

Accepted: 20 December 2017

Published: 16 January 2018

This article is part of the Thematic Series "Nanostructured liquid crystal systems and applications".

Guest Editor: A. Emelyanenko

(C) 2018 Maximean et al.; licensee Beilstein-Institut.

License and terms: see end of document.

\begin{abstract}
A new bisimidazolium salt with dodecyl sulfate as counterion has been designed and prepared. This salt shows a SmA phase that is stable at room temperature. The new ionic liquid crystal (ILC) was characterized by ${ }^{1} \mathrm{H} N M R,{ }^{13} \mathrm{C}$ NMR and IR spectroscopy. Its liquid crystalline properties were analyzed by polarizing optical microscopy (POM), differential scanning calorimetry (DSC) and powder X-ray diffraction (XRD) studies. The dielectric spectra of the ILC doped with different concentrations of carbon nanotubes (CNT) were recorded over a wide frequency and temperature range of $10^{-1}$ to $10^{7} \mathrm{~Hz}$ and $293-338 \mathrm{~K}$, respectively. The values of the activation energy were found in the range of $0.46-0.61 \mathrm{eV}$; the characteristic time was obtained by fitting the spectra of the dielectric loss with the Havriliak-Negami functions. As a result of doping the ILC with CNT, the electric conductivity increases significantly. Ionic conductivity is dominant and it was indirectly observed through the electrode polarization (EP) effect. The very high dielectric permittivity values and the decrease of the electric conductivity at low frequencies confirm the presence of EP.
\end{abstract}

\section{Introduction}

Ionic liquid crystals (ILCs) represent a very appealing class of materials that has found various recent applications in dyesensitized solar cells, battery materials, electrochemical sensors or energy storage devices. Their interesting properties result from the combination of liquid crystal (LC) and ionic liquid (IL) properties. The recent progress and development in the field of ILCs were reviewed in several publications [1-3]. There is a growing interest in ILs based on imidazolium cations. Particular attention is paid to the flexibly or rigidly linked bisimidazolium salts, the so-called gemini ILs. Gemini ILs can show interesting surfactant and liquid crystalline properties [4-12] and such salts were used in many applications, ranging from catalysis [13] to biological [14-16] or biochemical applications [17-19]. Recently, we have shown that it is possible to exchange 
the smaller anions $\left(\mathrm{Br}^{-}\right)$with alkyl sulfate ions to yield new ILCs based on bisimidazolium salts with flexible methylene spacer and long alkyl tails [20]. The LC properties are influenced, in the order of impact, by spacer length, alkyl tail length and, finally, by the length of alkyl chains attached to the sulfate groups [21]. Furthermore, the liquid crystalline compounds with alkyl sulfate anions have lower melting and clearing points. Hence, there is the interest to design such materials for further electro-optical applications [22-32].

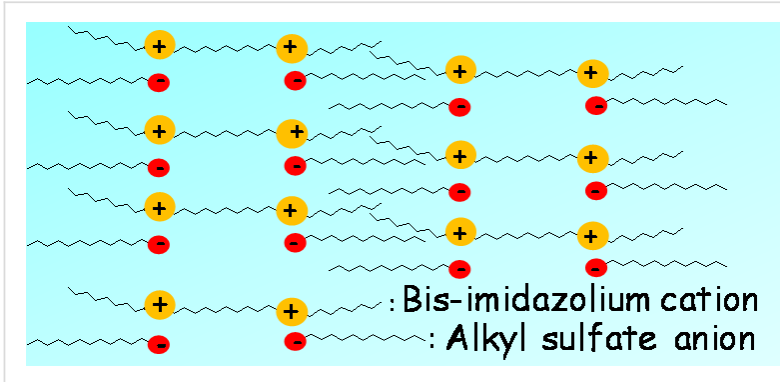

Figure 1: Schematic representation of the bilayer interdigitated SmA phase of the ILC based on bisimidazolium salts with alkyl sulfate ion.

Carbon nanotubes (CNTs) and nanoparticles were also dispersed in LCs [33-36]. It is well-known that the order of the LCs can be imposed on the CNTs in such a way that the alignment axis of the CNTs is driven by the LC reorientation controlled by an electric field [37]. The concentration and the spatial distribution of charges in the LC matrix will be affected by the presence of CNTs and, hence, the conductivity will be changed [38-44].

There are studies dedicated to the dielectric spectroscopy (DS) of ILCs doped with CNT [45]. In the present work we studied the effect of added CNTs on the dielectric properties of a new ILC based on a bisimidazolium salt with a dodecyl sulfate ion. The dielectric spectra of pure and CNT-doped ILC were recorded in the frequency range from $10^{-1}$ to $10^{7} \mathrm{~Hz}$ and in the temperature range from 293 to $338 \mathrm{~K}$ corresponding to the different phases of the ILC (mesophase and isotropic state). The values of the permittivity, dielectric loss and conductivity were deduced from the dielectric studies. The activation energy was calculated by employing the Vogel-Fulcher-Tammann law while the characteristic time was obtained by fitting the spectra of the dielectric loss with the Havriliak-Negami functions.

\section{Results and Discussion Synthesis of the bisimidazolium salt}

The preparation of new bisimidazolium salt with dodecyl sulfate anion employed in this study, along with the numbering scheme of intermediates, is presented in Scheme 1.

In the first step 1,1'-(1,10-hexanediyl)bisimidazole (1) was prepared starting from imidazole and 1,10-dibromodecane, as described by Bara and co-workers [7]. The bromide salt 2 was prepared by alkylation of $\mathbf{1}$ with 1-bromooctane in acetonitrile under reflux. The product was precipitated with ethyl ether, and purified by several recrystallizations from dichloromethane/ ethyl ether. The metathesis reaction of the bromide anion with sodium dodecyl sulfate, $\mathrm{C}_{12} \mathrm{H}_{25} \mathrm{OSO}_{3} \mathrm{Na}$, yielded the corresponding salt $\left[\mathrm{bisC}_{8} \mathrm{ImC}_{10}\right]\left[\mathrm{C}_{12} \mathrm{H}_{25} \mathrm{OSO}_{3}\right]_{2}$ (3). Compound 3 was characterized by several physico-chemical techniques, such as elemental analysis $(\mathrm{C}, \mathrm{H}, \mathrm{N}), \mathrm{IR},{ }^{1} \mathrm{H}$ and ${ }^{13} \mathrm{C}$ NMR spectroscopy, supporting the proposed structure. The exchange of bromide anion with dodecyl sulfate ion was easily confirmed by IR spectroscopy. The IR spectrum of $\left[\right.$ bisC $\left._{8} \mathrm{ImC}_{10}\right]\left[\mathrm{C}_{12} \mathrm{H}_{25} \mathrm{OSO}_{3}\right]_{2}$ shows a strong band at $1225 \mathrm{~cm}^{-1}$ assigned to the sulfate group.<smiles></smiles>

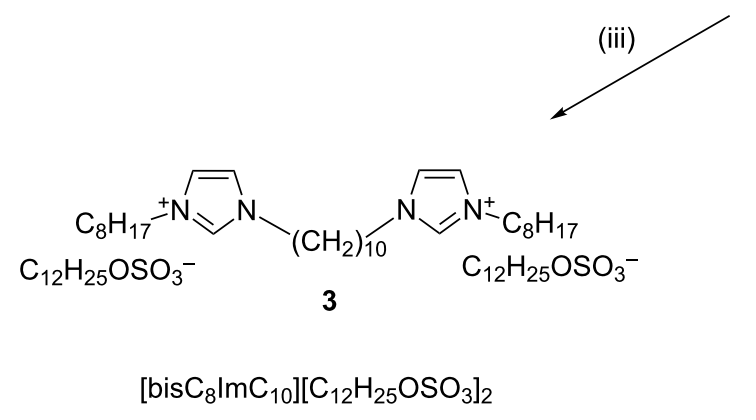

Scheme 1: Synthesis of bisimidazolium salt with dodecyl sulfate anion: (i) $\mathrm{Br}\left(\mathrm{CH}_{2}\right){ }_{10} \mathrm{Br}$, $\mathrm{NaH}$; (ii) $\mathrm{C}_{8} \mathrm{H}_{17} \mathrm{Br}$, acetonitrile; (iii) $\mathrm{C}_{12} \mathrm{H}_{25} \mathrm{OSO}{ }_{3} \mathrm{Na}$, methanol/ dichloromethane. 
Moreover, the ${ }^{1} \mathrm{H}$ and ${ }^{13} \mathrm{C}$ NMR spectra give additional support for the exchange of bromide ions with dodecyl sulfate ions. The signals assigned to the three protons belonging to the two imidazolium rings are shifted upfield in the ${ }^{1} \mathrm{H}$ NMR spectrum of $\left[\right.$ bisC $\left._{8} \mathrm{ImC}_{10}\right]\left[\mathrm{C}_{12} \mathrm{H}_{25} \mathrm{OSO}_{3}\right]_{2}$ compared to their position in the ${ }^{1} \mathrm{H}$ NMR of the bromide salt $\mathbf{2}$. The most significant change was observed for the signal assigned to the proton adjacent to the two nitrogen atoms (Scheme 1). For the bromide salt 2 this signal is located at $10.42 \mathrm{ppm}$, while for the dodecyl sulfate salt [bisC $\left.\mathrm{ImC}_{10}\right]\left[\mathrm{C}_{12} \mathrm{H}_{25} \mathrm{OSO}_{3}\right]_{2}$ this signal is shifted to $9.58 \mathrm{ppm}$. It is well documented that the anion-cation interactions have a strong effect on the NMR chemical shifts of protons belonging to the imidazolium ring. The NMR signals are shifted downfield due to the presence of hydrogen-bonding interactions in imidazolium-based ILC systems [32,46-51].

\section{Polarized optical microscopy (POM)}

The LC phase of $\left[\mathrm{bisC}_{8} \mathrm{ImC}_{10}\right]\left[\mathrm{C}_{12} \mathrm{H}_{25} \mathrm{OSO}_{3}\right]_{2}$ was determined based on the POM observations. Two different pictures of the textures developed on cooling from the isotropic state are shown in Figure 2. These observations were confirmed later by
XRD studies. On cooling the sample from the isotropic state, typical fan-shape or focal conic textures together with several homeotropic areas were found, leading to an unambiguously assignment of a SmA phase. ILCs are well known to exhibit predominantly lamellar phases, with the SmA phase being the most common phase for such materials, in particular due to electrostatic interactions and ion-ion stacking in ILCs.

\section{Differential scanning calorimetry (DSC)}

The transitions and their corresponding temperatures together with the enthalpy values associated to these processes are presented in Figure 3. The transitions of [bisC $\left.\mathrm{ImC}_{10}\right]-$ $\left[\mathrm{C}_{12} \mathrm{H}_{25} \mathrm{OSO}_{3}\right]_{2}$ between mesophase and isotropic state observed by DSC are broad, with $T_{\text {onset }}=321.15 \mathrm{~K}$. The temperature range of the liquid crystalline phase is limited to approx. $10 \mathrm{~K}$ during the heating run. The first heating run of the bisimidazolium salt shows a broad peak including the transition from the crystalline state to the LC phase and the following transition to the isotropic state, giving only the combined enthalpy of the two processes. However, POM observations clearly indicated that the two transformations are well separated. The
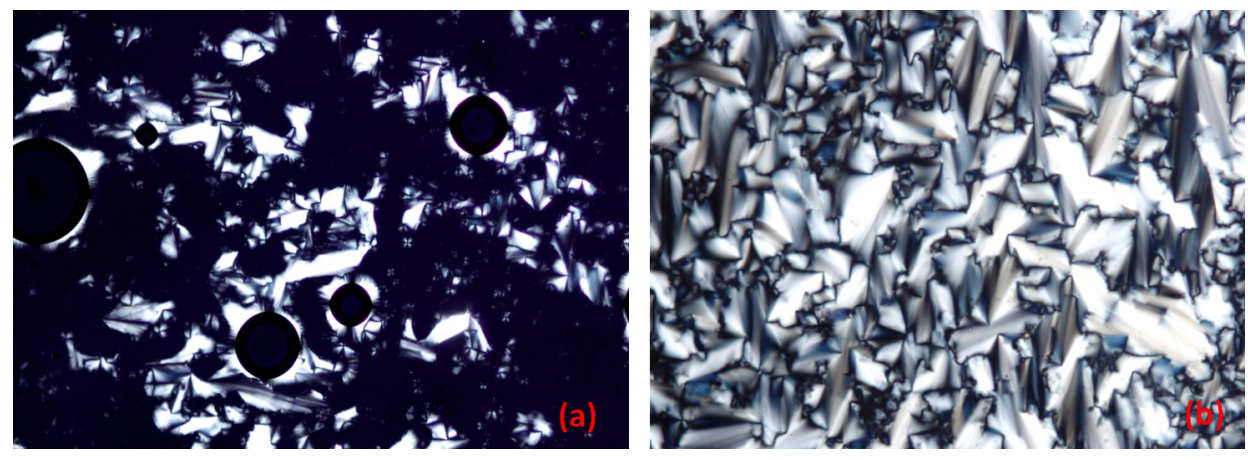

Figure 2: $\mathrm{POM}$ pictures of $\left[\mathrm{bisC}_{8} \mathrm{ImC}_{10}\right]\left[\mathrm{C}_{12} \mathrm{H}_{25} \mathrm{OSO}_{3}\right]_{2}$ on cooling from the isotropic state: at $318 \mathrm{~K}(\mathrm{a})$ and at $303 \mathrm{~K}(\mathrm{~b})$.
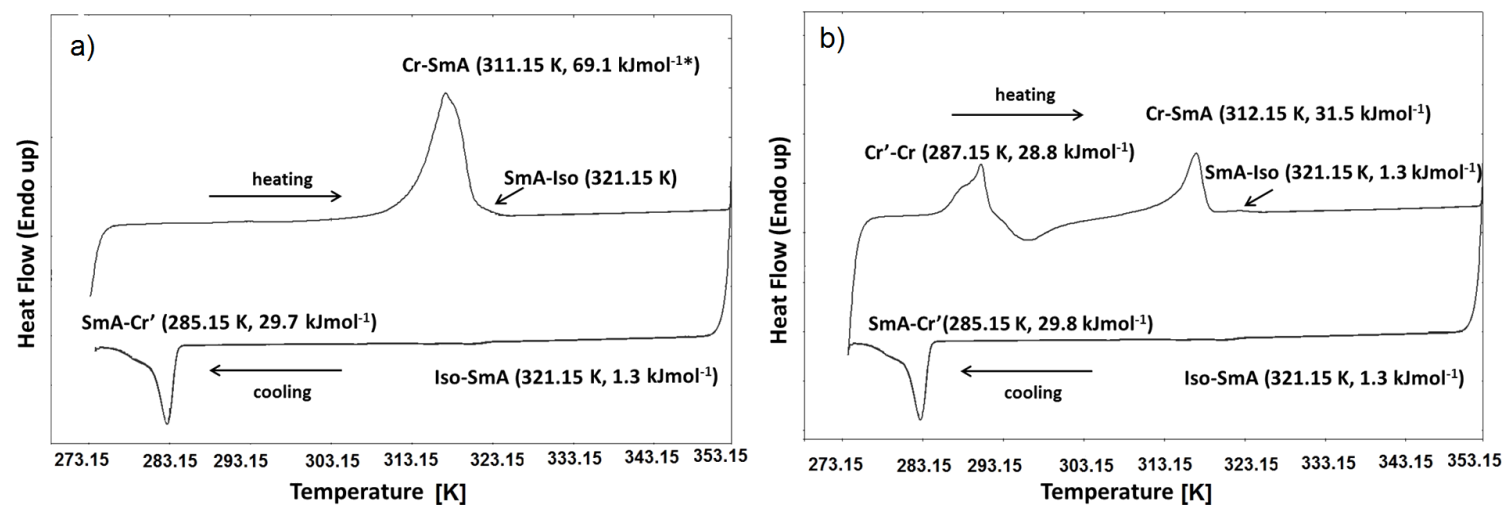

Figure 3: DSC traces for [ $\left.\mathrm{bisC}_{8} \mathrm{ImC} \mathrm{m}_{10}\right]\left[\mathrm{C}_{12} \mathrm{H}_{25} \mathrm{OSO}_{3}\right]_{2}$ : (a) first and (b) second heating-cooling cycle recorded in the temperature range of 273-353 $\mathrm{K}$ with a scan rate of $10 \mathrm{~K} / \mathrm{min}$. Thermal parameters are given in brackets. The given enthalpy contains the combined values for the two transitions Cr-SmA and SmA-Iso. 
following cooling run shows the Iso-SmA phase transition at $321.15 \mathrm{~K}$ followed by a second transition at $285.15 \mathrm{~K}$, which was assigned to a transition from the LC phase to a different crystalline phase $\left(\mathrm{Cr}^{\prime}\right)$. This second $\mathrm{Cr}^{\prime}$ phase is not thermodynamically stable at room temperature and it transitions to the first crystalline phase at $287.15 \mathrm{~K}$ during the subsequent heating run (in fact, it is a melting transition followed by the cold crystallization to the $\mathrm{Cr}$ phase). The following heating-cooling cycles are perfectly reproducible with the two transitions $\mathrm{Cr}-\mathrm{SmA}$ and SmA-Iso well separated during the heating runs (Figure 3b). Importantly, POM observations show that on cooling from the isotropic state, the SmA phase is stable down to room temperature over time with a slow crystallization occurring over the course of hours based on POM observations. Obviously, the stability domain of the SmA phase is higher (ca. $36 \mathrm{~K}$ ) during the cooling step, allowing for precise dielectric measurements in the LC phase.

\section{Powder X-ray diffraction measurements}

The nature of the LC phase was unequivocally confirmed by powder X-ray diffraction measurements. The XRD measurements were performed at $298 \mathrm{~K}$ after the sample was previously heated at $333 \mathrm{~K}$ to reach the isotropic state and then cooled down to room temperature in the mesophase temperature domain. The results are presented in Figure 4. The XRD pattern of $\left[\right.$ bisC $\left._{8} \mathrm{ImC}_{10}\right]\left[\mathrm{C}_{12} \mathrm{H}_{25} \mathrm{OSO}_{3}\right]_{2}$ salt shows one sharp and intense diffraction peak in the small-angle region $(27.3 \AA)$ and a second broad peak at wide angles (around $4.5 \AA$ ). The first peak was assigned to the (001) reflection corresponding to a lamellar structure while the broad peak is due to the liquidlike order of the molten alkyl chains.

The results of a simple molecular calculation based on an alltrans extended model revealed a molecular length of about $39 \AA$. Indeed, by comparison of the experimental value of the layer thickness $(27.3 \AA)$ with the molecular length resulting from molecular calculations, it is justified to assume an interdigitated double layer structure for the SmA mesophase $[4,20]$.

\section{Dielectric spectroscopy}

The DS measurements were performed both for the pure ILC and for various mixtures of CNT-doped ILC (concentration $0.05 \% \mathrm{w} / \mathrm{w}$ and $0.5 \% \mathrm{w} / \mathrm{w}$ ) in the frequency range from $10^{-1}$ to $10^{7} \mathrm{~Hz}$. The temperature range was chosen in agreement with the DSC and the POM observations for the phase transitions, between 293 and $338 \mathrm{~K}$.

The logarithmic permittivity for the pure ILC and the CNTdoped ILC as a function of the temperature is presented in Figure 5. It was found that the permittivity has higher values for the CNT-doped ILC and, in the range of 320-338 K, the plots of the permittivity for the $0.05 \% \mathrm{CNT}$ and $0.5 \% \mathrm{CNT}$ concentrations overlap.

The temperature variation of the dielectric loss for the pure ILC and the CNT-doped ILC is shown in Figure 6. It was found that the dielectric loss increases with the temperature and with the CNT concentration.

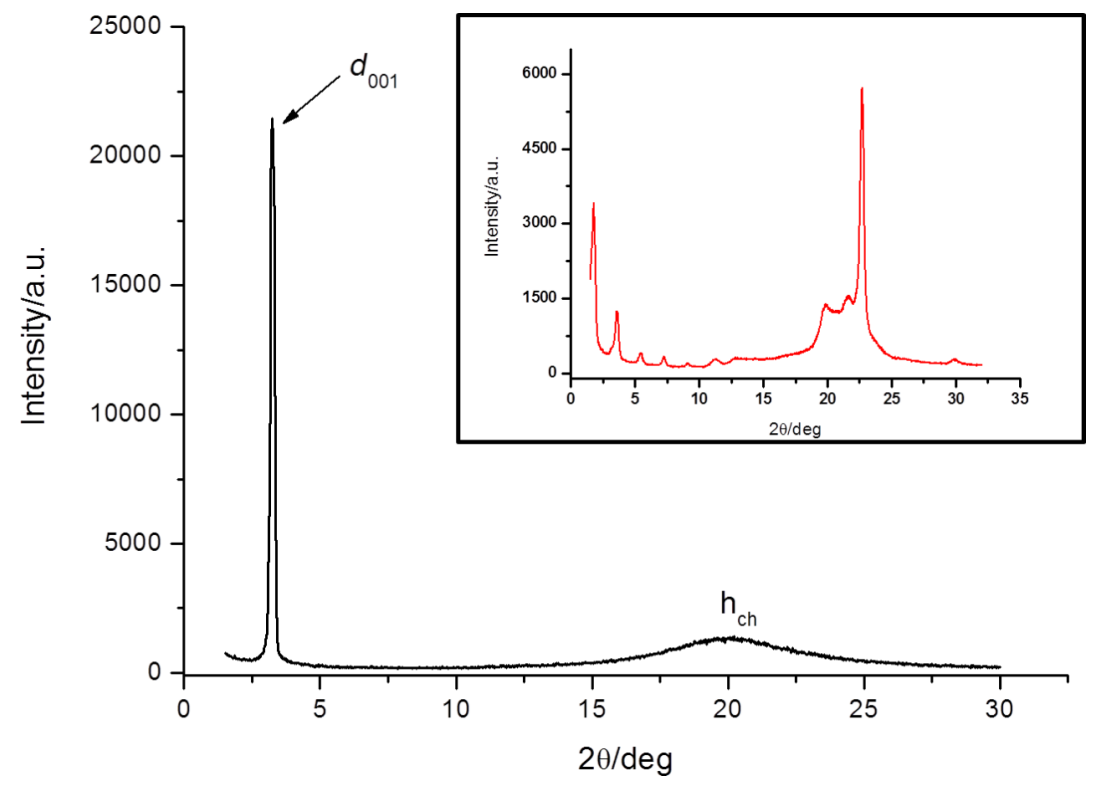

Figure 4: Powder XRD pattern of [ $\left.\mathrm{bisC}_{8} \mathrm{ImC}_{10}\right]\left[\mathrm{C}_{12} \mathrm{H}_{25} \mathrm{OSO}_{3}\right]_{2}$ recorded at $298 \mathrm{~K}$, after cooling from the isotropic state. Inset: the XRD pattern recorded at $298 \mathrm{~K}$ in the crystalline state (prior to heating). 


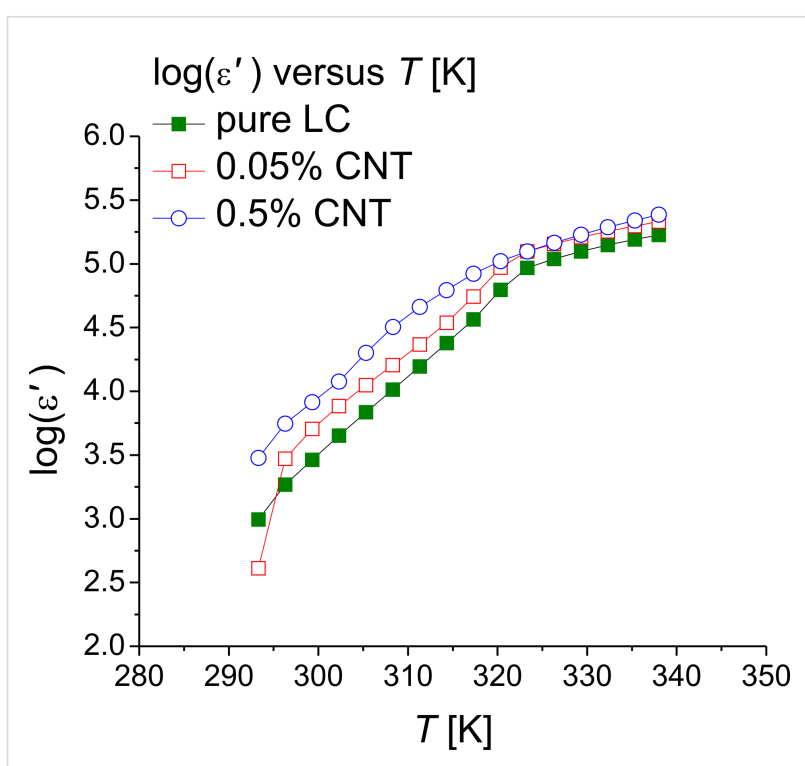

Figure 5: Permittivity as a function of the temperature (logarithmic scale) for pure ILC (green solid squares), ILC doped with $0.05 \%$ CNTs (red open squares) and ILC doped with $0.5 \%$ CNTs (blue open circles).

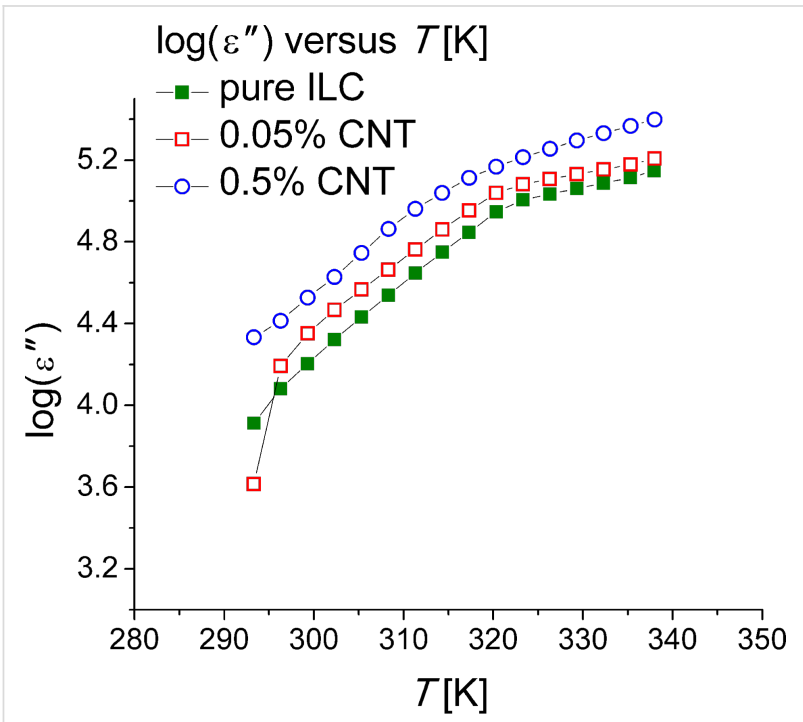

Figure 6: Dielectric loss as a function of the temperature (logarithmic scale) for pure ILC (green solid squares), ILC doped with $0.05 \%$ CNTs (red open squares) and ILC doped with $0.5 \%$ CNTs (blue open circles).

The characteristic relaxation times were obtained by fitting the spectra of the dielectric loss with the Havriliak-Negami (HN) function [52]:

$$
\varepsilon_{\mathrm{HN}}^{*}(\omega)=\varepsilon^{\prime}(\omega)-\mathrm{i} \varepsilon^{\prime \prime}(\omega)=\varepsilon_{\infty}+\frac{\varepsilon_{\mathrm{LF}}-\varepsilon_{\infty}}{\left(1+\left(\mathrm{i} \omega \cdot \tau_{\max }\right)^{\alpha}\right)^{\beta}}
$$

with $0<\alpha<1$ and $0<\beta<1$, where $\varepsilon^{\prime}(\omega)$ is the permittivity and $\varepsilon^{\prime \prime}(\omega)$ is the dielectric loss, $\varepsilon_{\mathrm{LF}}$ is the low-frequency (LF) permittivity and $\varepsilon_{\infty}$ is the permittivity in the high-frequency (HF) limit and $\tau_{\max }$ is the characteristic relaxation time of the dielectric relaxation process.

The dependency $\tau_{\max }=f(1 / T)$ can be modeled using the Vogel-Fulcher-Tammann (VFT) law, as follows:

$$
\tau_{\max }=\tau_{\infty} \exp \left[\frac{A}{k_{\mathrm{B}}\left(T-T_{\mathrm{V}}\right)}\right]
$$

where $A$ is a material constant, $k_{\mathrm{B}}$ is Boltzmann's constant, $T$ is the temperature, $T_{\mathrm{V}}$ is the Vogel temperature and $\tau_{\infty}$ is a preexponential factor.

Figure 7 presents the characteristic relaxation time as a function of the inverse temperature for the pure ILC and the CNTdoped ILC. For the pure ILC and for the lower CNT doping concentrations, there are two slopes, attributed to the isotropic and the SmA phases. At the higher CNT concentration (0.5\%) the transition isotropic-SmA could not be detected clearly, probably due to the very low energies involved in the transition. In the isotropic phase, the curves of the relaxation time for the $0.05 \% \mathrm{CNT}$ and $0.5 \% \mathrm{CNT}$ concentrations overlap, and for lower temperatures, the relaxation time decreases with the CNT concentration. The pure ILC has a relaxation time higher than the doped ILC in the temperature range of the mesophase.

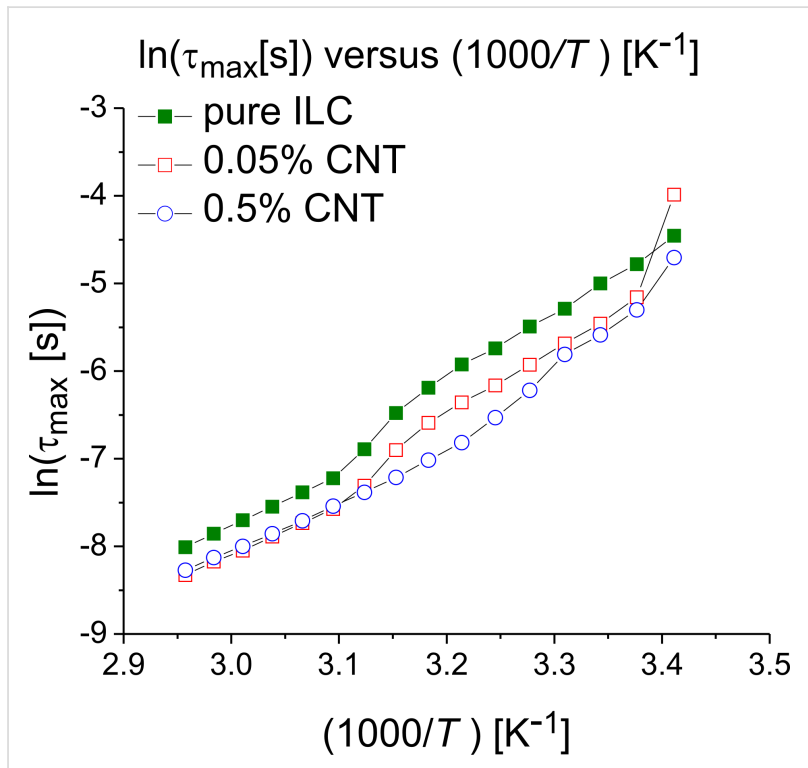

Figure 7: Relaxation time as function of the inverse temperature (logarithm scale) for pure ILC (green solid squares), ILC doped with $0.05 \%$ CNTs (red open squares) and ILC doped with $0.5 \%$ CNTs (blue open circles). 
Table 1 gives the values of the activation energy and the relaxation times in the corresponding temperature domains. The following observations can be made according to data presented in Table 1: a) the activation energy increases with CNT concentration; b) for the same CNT concentration, the activation energy is smaller in the isotropic phase (at higher temperatures).

There are "jumps" in the characteristic relaxation time around certain temperatures such as 293-296 K for the CNT-doped samples and $314.15-323.15 \mathrm{~K}$ for the pure ILC and the $0.05 \%$ CNT doped sample (Figure 7). These jumps can be attributed either to the fitting procedure or to one or more phase transitions. The isotropic-SmA transition is found in the range of 314-323 K. In order to study this phenomenon, the permittivity and dielectric loss were plotted as functions of the frequency in the abovementioned temperature ranges. These curves are shown in Figure 8 and Figure 9. The ILC permittivity as a function of the frequency has three distinct slopes, one between $10^{-1}$ and $10^{2} \mathrm{~Hz}$, one between $10^{2}$ and $10^{4} \mathrm{~Hz}$ and the third one between $10^{4}$ and $10^{7} \mathrm{~Hz}$. This type of dependence also occurs for the CNT doping concentrations of $0.05 \%$ and $0.5 \%$.

The dielectric loss of the ILC can be divided in two regions, between $10^{-1}$ and $10^{2} \mathrm{~Hz}$ and between $2 \cdot 10^{2}$ and $10^{7} \mathrm{~Hz}$ (Figure 8). At lower temperatures, for the CNT- doped samples (Figure 9a,b), the straight lines with the greater slope are seen over a wider frequency range (more evident in Figure 9c, at 293 K). In the dielectric loss spectra, presented in Figure 8 and Figure 9, a dipolar relaxation process was identified in the range of $10^{2}-10^{3} \mathrm{~Hz}$, with only the descendent slope being observed. The Havriliak-Negami fitting function (Equation 1) are centered on this process. In the fitting procedure the exponent $\beta$ was set to 1 , and the other parameters were left optimized by fitting the $\mathrm{HN}$ function to the experimental data. It was found that the permittivity and the dielectric loss values increase with the temperature for pure ILC and for all CNT-doped samples.

The permittivity and the dielectric loss have high values in the LF domain, both for the pure and the doped-ILC. The high values in the range of $10^{6}-10^{8} \mathrm{~Hz}$ are due to the presence of

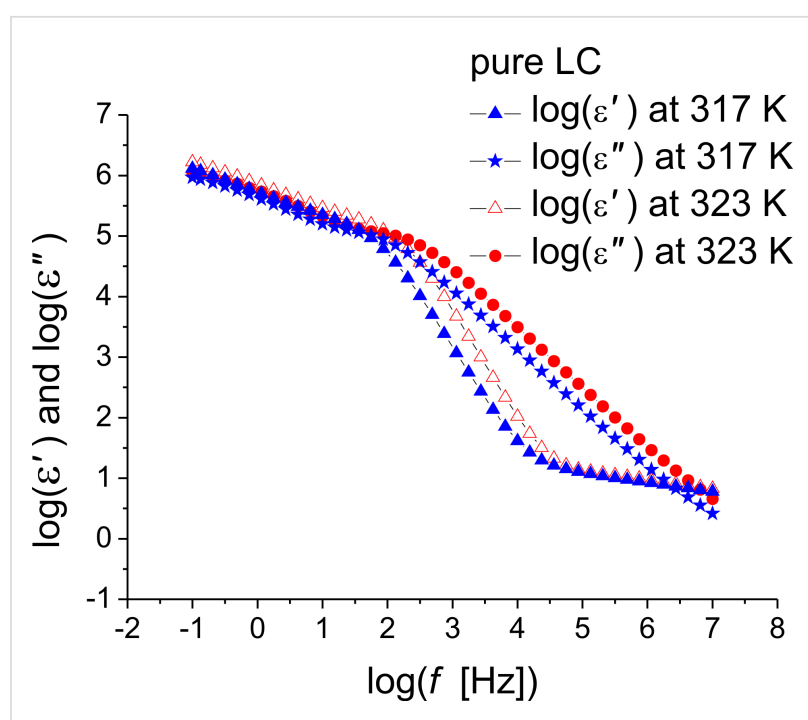

Figure 8: Permittivity and dielectric loss as functions of the frequency (logarithmic scale) for the pure ILC at two constant temperatures, 317 and $323 \mathrm{~K}$.

free ions. Figure 10 shows the variation of the real part of the conductivity as a function of the temperature for the ILC and the CNT-doped ILC. A linear dependency is observed, the change of the slope being attributed to the different phases. This behavior is more clearly observed for the ILC (solid squares) and the ILC doped with $0.05 \%$ CNTs (open squares). The conductivity increases with the CNT concentration.

Figure 11 shows the real part of the conductivity of the pure ILC as a function of the frequency (logarithmic scale), at three different constant temperatures. An increase of the conductivity with CNT concentration and temperature is also observed as presented in Figure 12.

\section{Ionic conductibility}

Two major polarizing mechanisms can be studied by means of DS: a) polarization due to orientation of permanent molecular electrical dipoles and b) polarization due to the movement of mobile charge carriers. Due to the presence of mobile charge carriers in LCs, a supplementary induced polarization needs to

\begin{tabular}{|c|c|c|c|c|}
\hline no. & sample type & temperature domain $[\mathrm{K}]$ & activation energy, $E_{\mathrm{a}}{ }^{\mathrm{a}}[\mathrm{eV}]$ & relaxation time, $\mathrm{T}_{\max }[\mathrm{s}]$ \\
\hline \multirow[t]{2}{*}{1} & pure ILC & $296-317$ & 0.61 & $5.500 \times 10^{-13}$ \\
\hline & & $323-338$ & 0.49 & $1.700 \times 10^{-11}$ \\
\hline \multirow[t]{2}{*}{2} & ILC doped with $0.05 \%$ CNT & $293-317$ & 0.59 & $1.169 \times 10^{-13}$ \\
\hline & & $323-338$ & 0.47 & $2.364 \times 10^{-11}$ \\
\hline 3 & ILC doped with $0.5 \%$ CNT & $309-338$ & 0.46 & $3.105 \times 10^{-11}$ \\
\hline
\end{tabular}

an these calculations, the Vogel temperature was considered zero in the initial fitting. Hence, the VFT equation approximates an Arrhenius-like expression, the material constant $A$ being incorporated into the activation energy $E_{\mathrm{a}}$. 


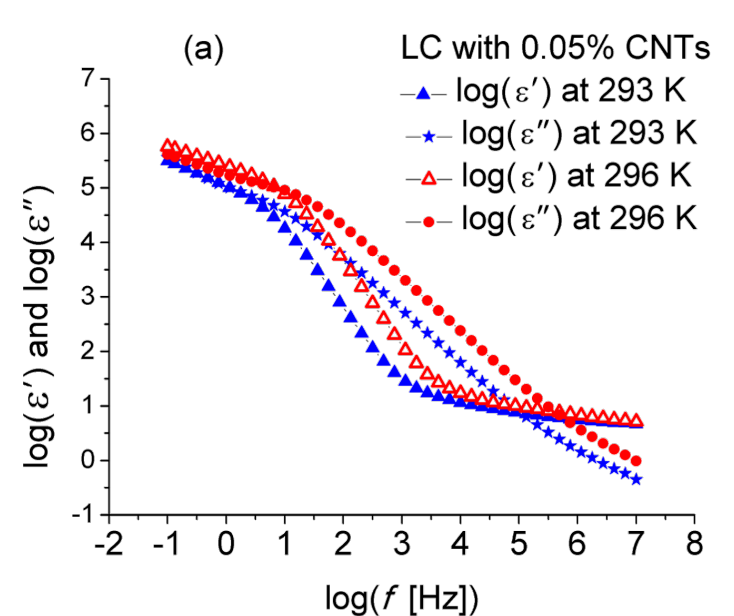

(c) LC with $0.5 \%$ CNTs

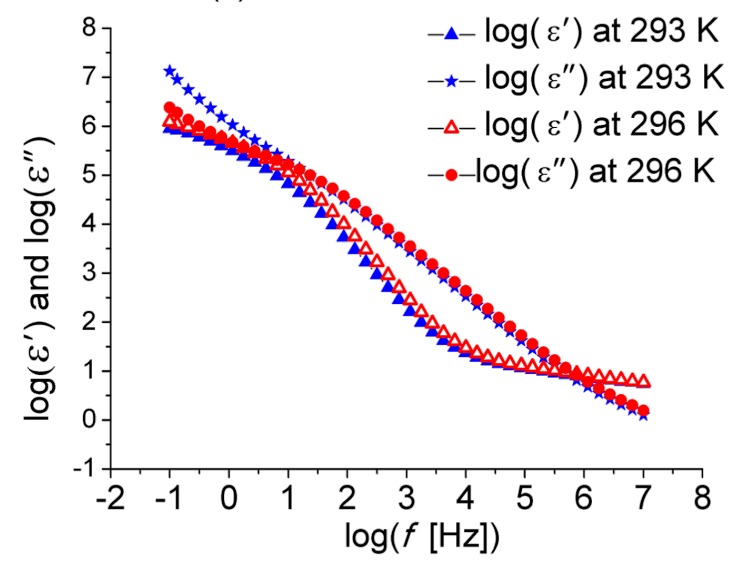

(b) LC with $0.05 \%$ CNTs

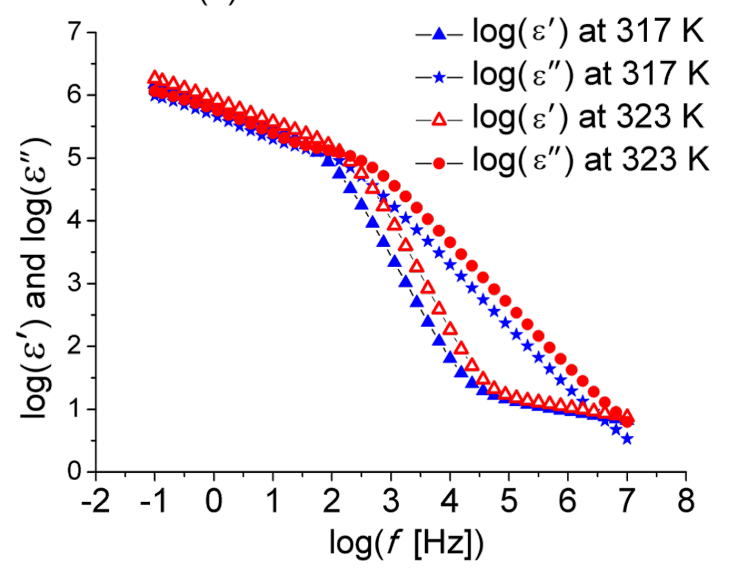

(d) LC with $0.5 \%$ CNTs

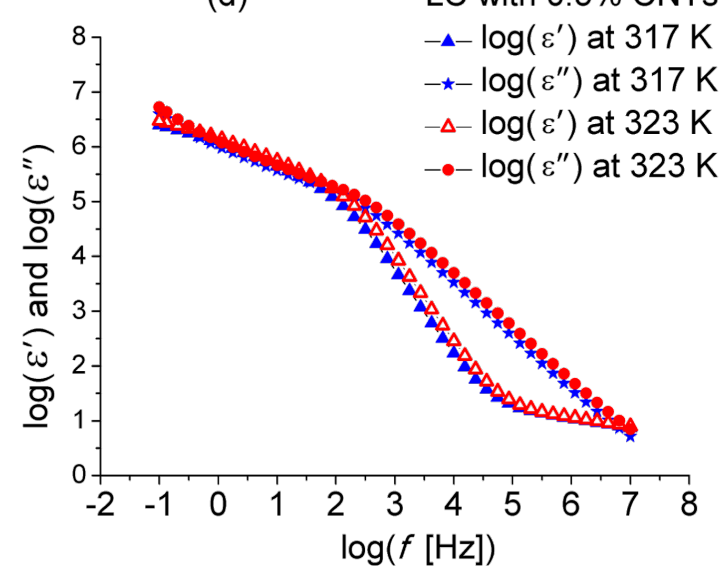

Figure 9: Permittivity and dielectric loss as functions of the frequency (logarithmic scale) at different constant temperatures for (a,b) ILC with $0.05 \%$ CNTs, and (c,d) ILC with $0.5 \%$ CNTs.

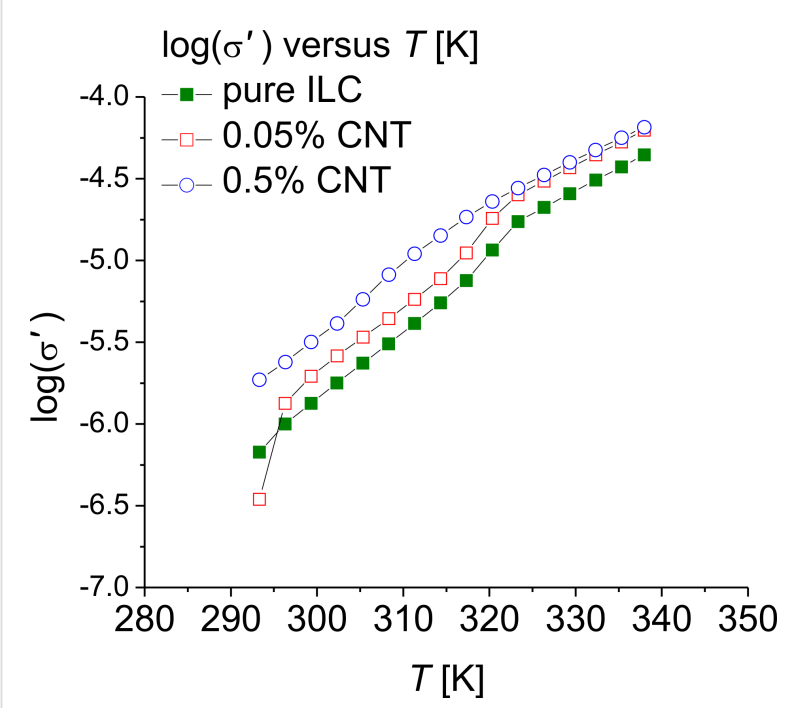

Figure 10: Real part of the conductivity versus temperature for pure ILC and ILC doped with CNTs, at $10 \mathrm{kHz}$.

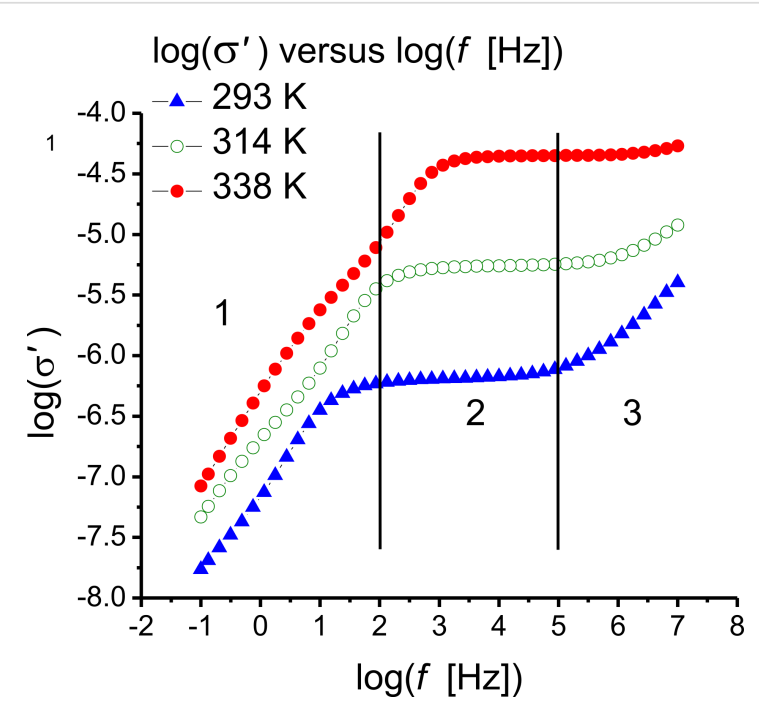

Figure 11: Real part of the conductivity as a function of the frequency (logarithmic scale) for the pure ILC, at three different constant temperatures. Region 1: low and very low frequency; region 2: medium frequency; region 3: high frequency. 


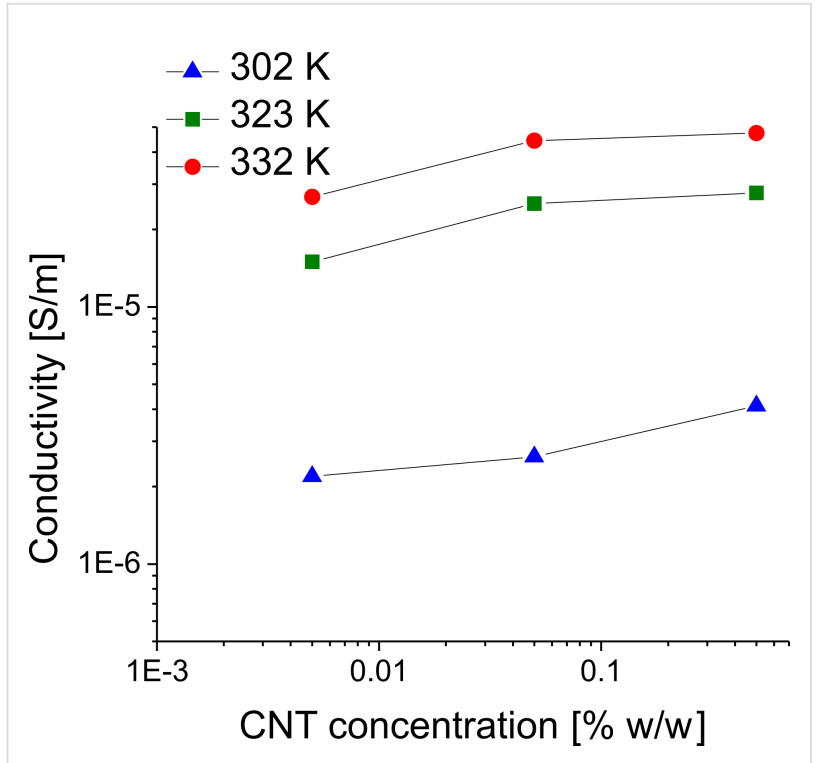

Figure 12: Conductivity as a function of the CNT concentration at three constant temperatures.

be considered when interpreting experimental data, namely the electrode polarization, resulting from charge accumulation at the electrode/sample interface.

Experimental data can be presented either by means of the complex dielectric function or of electric conductivity. The AC conductivity $\sigma^{*}(\omega)$ is a complex function:

$$
\sigma^{*}(\omega)=\sigma^{\prime}(\omega)+\mathrm{i} \sigma^{\prime \prime}(\omega)
$$

In agreement with Maxwell's equations, a direct general relationship can be established between the electrical conductivity and the dielectric permittivity of a medium. For a sinusoidal electric field Equation 3 becomes:

$$
\sigma^{*}(\omega)=\sigma_{0}+\mathrm{i} \omega \varepsilon_{0} \varepsilon *(\omega)
$$

The real and the imaginary parts are $\sigma^{\prime}(\omega)=\sigma_{0}+\omega \varepsilon_{0} \varepsilon^{\prime \prime}(\omega)$ and $\sigma^{\prime \prime}(\omega)=\omega \varepsilon_{0} \varepsilon^{\prime}(\omega)$, respectively.

As shown in Figure 11, at medium frequencies $\left(10^{3}-10^{5} \mathrm{~Hz}\right)$, the ac conductivity and permittivity spectra are controlled by ion movements in the bulk of the electrolyte. At low frequencies $\left(10^{-1}-10^{3} \mathrm{~Hz}\right)$, approximately region 1 in Figure 11, the behavior is controlled by "electrode polarization" effects. Thus, the electric conductivity decreases significantly when the frequency decreases. In the frequency range below $100 \mathrm{MHz}$, the ionic conductivity spectra obey the Jonscher power law $[52,53]$

$$
\sigma^{\prime}(\omega)=\sigma_{\mathrm{dc}}+K_{\mathrm{c}} \omega^{1-N}
$$

where $0<N \leq 1$ and $\sigma_{\mathrm{dc}}$ is the dc conductivity (usually $0.1 \leq N \leq 0.4)$. The parameter $\sigma_{\mathrm{dc}}$ is obtained from the electrical conductivity spectra by extrapolation to the $\omega \rightarrow 0$ limit.

For samples with ionic carriers, obtaining the conductivity values, $\sigma_{\mathrm{dc}}$, is not trivial $[38,54,55]$. In this case the effects of the electrode polarization and of the ionic conductivity overlap at medium and low frequencies in the conductivity spectra (Figure 11 ). The electric conductivity is $\sigma_{\mathrm{dc}}=q \cdot \mu \cdot n$, where $q$ is the electrical charge (in C), $n$ is the concentration (in $\mathrm{cm}^{-3}$ ), and $\mu$ is the mobility (in $\mathrm{cm}^{2} \cdot \mathrm{s}^{-1} \cdot \mathrm{V}^{-1}$ ).

The bulk electrical ionic conductivity $\sigma_{\mathrm{dc}}$ obeys the Arrhenius law [5]:

$$
\sigma_{\mathrm{dc}}=\frac{B}{T} \exp \left(-\frac{W_{\mathrm{A}}}{k_{\mathrm{B}} T}\right)
$$

where $T$ is the absolute temperature, $B$ is a pre-exponential constant of the material and $W_{\mathrm{A}}$ is a constant associated with the activation energy. Usually, the activation energy includes the energy terms for the formation and migration of ions.

The experimental results showed that the real part of the permittivity, $\varepsilon^{\prime}$, representing the dielectric conductivity, increases with the increase of CNT concentration; the same behavior was seen for the imaginary part of the permittivity, $\varepsilon^{\prime \prime}$, representing the dielectric loss. The permittivity being linked to dipole movements, an increase of the CNT concentration will lead to possible sample heating. Moreover, the ionic conductivity, $\sigma$, increases with CNT concentration (Figure 12). In this way, additional heating results from an increase of the CNT concentration, this process being frequency dependent.

\section{Conclusion}

A new ILC based on bisimidazolium salt with a spacer of ten carbon atoms and octyl tails and dodecyl sulfate as counterion was synthesized. The SmA phase was unambiguously assigned based on polarized optical microscopy observations and XRD studies while the corresponding phase transition enthalpies were obtained by DSC. The ILC was doped with CNT in concentration of $0.05 \% \mathrm{w} / \mathrm{w}$ and $0.5 \% \mathrm{w} / \mathrm{w}$. The dielectric spectra were recorded in the frequency range from $10^{-1}$ to $10^{7} \mathrm{~Hz}$. The dependence of the dielectric constant and electric energy loss on frequency and temperature was studied. The activation energy was determined and the characteristic time was obtained by fitting the spectra of the dielectric loss with the 
Havriliak-Negami function. The study of the dielectric spectra leads to the following observations: (1) The study of the dielectric permittivity and electric conductivity spectra is complex, due to the superposition of ionic conductivity effect and dipolar relaxation specific to LC. Ionic conductivity is dominant and its effects are indirectly seen through the electrode polarization (EP) effect. (2) The very high dielectric permittivity values and the decrease of the electric conductivity at low frequencies confirm the presence of EP. (3) The doping with CNTs results in an increase of the conductivity. (4) Relaxation times decrease with CNT concentration. (5) In the lower temperature range, the presence of CNTs leads to a decrease of the activation energy while for higher temperatures, the activation energies are very similar for the pure ILC and the doped samples. As expected, the activation energies are lower at higher temperatures.

These preliminary studies revealed an interesting influence of the CNT concentration on the dielectric behavior of the bisimidazolium salt. This investigation will be extended to new ionic liquid crystals with a large range of doping concentration in order to complete the existing image on these aspects. Special attention will be paid to low-frequency studies of the effects related to very low CNT concentration.

\section{Experimental}

\section{Characterization methods}

All chemicals were used as supplied. C, H, N analyses were carried out with an EuroEA 3300 instrument. IR spectra were recorded on a Bruker spectrophotometer using $\mathrm{KBr}$ discs or by using a Jasco FTIR 4200 spectrophotometer coupled to an ATR PIKE GladiATR device. ${ }^{1} \mathrm{H}$ and ${ }^{13} \mathrm{C}$ NMR spectra were recorded on a Bruker spectrometer operating at $500 \mathrm{MHz}$, using $\mathrm{CDCl}_{3}$ as solvent. ${ }^{1} \mathrm{H}$ chemical shifts were referenced to the solvent peak position, $\delta=7.26 \mathrm{ppm}$. The phase assignment for the bisimidazolium salt was evaluated by polarizing optical light microscopy (POM) [56,57], placed on untreated glass slides, using a Nikon 50iPol microscope equipped with a Linkam THMS600 hot stage and TMS94 control processor. Temperatures and enthalpies of transitions were recorded by using differential scanning calorimetry (DSC) technique employing a Diamond DSC Perkin Elmer instrument. The bisimidazolium salt was studied at a scanning rate of $10 \mathrm{~K} / \mathrm{min}$ after being encapsulated in an aluminum pan. Three heating-cooling cycles were performed for this sample.

\section{Synthesis of $\left[\mathrm{bisC}_{8} \mathrm{ImC} \mathrm{C}_{10}\right]\left[\mathrm{C}_{12} \mathrm{OSO}_{3}\right]_{2}$}

A solution of sodium dodecyl sulfate $(2.18 \mathrm{~g}, 7.5 \mathrm{mmol})$ in methanol $(60 \mathrm{~mL})$ was added dropwise to a solution of compound $2(2 \mathrm{~g}, 3.0 \mathrm{mmol})$ in dichloromethane $(50 \mathrm{~mL})$. The mixture was stirred at room temperature for $1 \mathrm{~h}$ after which $100 \mathrm{~mL}$ of deionised water was added. The organic layer was separated and washed repeatedly with water until no reaction with silver nitrate for $\mathrm{Br}^{-}$was noticed. The organic phase was dried over sodium sulfate followed by solvent removal with a rotary evaporator. The product was recrystallized twice from a mixture of dichloromethane and ethyl ether to yield an off-white waxy solid. Yield $75 \%$, off-white waxy solid. Anal. calcd for $\mathrm{C}_{56} \mathrm{H}_{110} \mathrm{~N}_{4} \mathrm{O}_{8} \mathrm{~S}_{2}$ : C, 65.20; H, 10.75; N, 5.43; found: C, 65.59; $\mathrm{H}, 11.03$; N, 5.27; ${ }^{1} \mathrm{H}$ NMR $\left(500 \mathrm{MHz}, \mathrm{CDCl}_{3}\right) \delta 9.58(\mathrm{~s}, 2 \mathrm{H})$, $7.78(\mathrm{~s}, 2 \mathrm{H}), 7.24(\mathrm{~s}, 2 \mathrm{H}), 4.32-4.20(\mathrm{~m}, 8 \mathrm{H}), 4.01(\mathrm{t}, 4 \mathrm{H}), 1.94-$ $1.84(\mathrm{t}, 8 \mathrm{H}), 1.64(\mathrm{t}, 4 \mathrm{H}), 1.42-1.22(\mathrm{~m}, 68 \mathrm{H}), 0.86(\mathrm{t}, 12 \mathrm{H})$; ${ }^{13} \mathrm{C}$ NMR $\left(125 \mathrm{MHz}, \mathrm{CDCl}_{3}\right) \delta 136.9,123.3,121.6,67.8,49.9$, 49.4, 31.9; 29.7, 29.6, 29.5, 29.4, 22.7, 14.1; IR (ATR, $\mathrm{cm}^{-1}$ ): 3136, 3109, 2957, 2919, 2851, 1568, 1467, 1379, 1225, 1169, $1063,1044,1005,932,792,723,623,580$.

\section{Preparation of the mixtures of LC doped with CNTs}

Single-walled CNTs (Aldrich code 519308) with a diameter between 1.2 and $5 \mathrm{~nm}$ were employed in this study. Two $\mathrm{CNT} /\left[\right.$ bisC $\left._{8} \mathrm{ImC}_{10}\right]\left[\mathrm{C}_{12} \mathrm{H}_{25} \mathrm{OSO}_{3}\right]_{2}$ mixtures with different amounts of CNTs $(0.5 \% \mathrm{w} / \mathrm{w}$ and $0.05 \% \mathrm{w} / \mathrm{w})$ were prepared for dielectric measurements by consecutive dilution of an initial sample containing $2 \%$ CNTs. The starting sample was prepared by dissolving first the bisimidazolium salt $(0.2 \mathrm{~g})$ in a minimum volume of dichloromethane $(1 \mathrm{~mL})$ followed by addition of CNT (0.004 g) [58]. The resulting mixture was sonicated for at least 60 min followed by the removal of the solvent, drying in vacuum and cooling at $0{ }^{\circ} \mathrm{C}$. All samples were kept at $0{ }^{\circ} \mathrm{C}$ before the physical measurements. Prior to dielectric measurements, the samples were heated to $50{ }^{\circ} \mathrm{C}$ and sonicated for at least 15 min to ensure an homogeneous dispersion of CNTs in the ionic liquid crystalline sample.

\section{X-ray diffraction}

The X-ray diffraction measurements were made on a D8 Advance diffractometer (Bruker AXS GmbH, Germany), in parallel beam setting, with monochromatized $\mathrm{Cu} \mathrm{K} \alpha_{1}$ radiation $(\lambda=1.5406 \AA)$, scintillation detector, and horizontal sample stage. The measurements were performed in symmetric $(\theta-\theta)$ geometry in the $2 \theta$ range from 1.5 to $30^{\circ}$ in steps of $0.02^{\circ}$, with measuring times per step in the range of 5-40 s. The sample was deposited on a $\mathrm{Si}(100)$ plate, heated to the isotropic state and then cooled down to room temperature prior to data acquisition

\section{Dielectric spectroscopy}

The dielectric spectroscopy measurements were performed using a broadband dielectric spectrometer, NOVOCONTROL, with an Alpha-A high-performance frequency analyzer in the LF domain (0.01 to $10^{7} \mathrm{~Hz}$ ), equipped with WinDETA soft- 
ware. The temperature was controlled within $0.2 \mathrm{~K}$, at a constant ac voltage of $0.5 \mathrm{~V}$.

\section{Acknowledgements}

Constantin Paul Ganea thanks the Romanian Ministry of Education and Research for financial support under PN16-480101 of CORE Program. Viorel Cîrcu acknowledges the funding from UEFISCDI, project number PN-II-ID-PCE-2011-3-0384. Doina Manaila Maximean thanks Ligia Frunza and Stefan Frunza for useful discussions.

\section{ORCID ${ }^{\circledR}$ iDs}

Viorel Cîrcu - https://orcid.org/0000-0001-9725-9577

\section{References}

1. Goossens, K.; Lava, K.; Bielawski, C. W.; Binnemans, K. Chem. Rev. 2016, 116, 4643-4807. doi:10.1021/cr400334b

2. Axenov, K. V.; Laschat, S. Materials 2011, 4, 206-259. doi: $10.3390 / \mathrm{ma} 4010206$

3. Cîrcu, V. Ionic Liquid Crystals Based on Pyridinium Salts. In Progress and Developments in Ionic Liquids; Handy, S., Ed.; InTech: Rijeka, Croatia, 2017; pp 285-311. doi:10.5772/65757

4. Yang, M.; Stappert, K.; Mudring, A.-V. J. Mater. Chem. C 2014, 2, 458-473. doi:10.1039/C3TC31368A

5. Robertson, L. A.; Schenkel, M. R.; Wiesenauer, B. R.; Gin, D. L. Chem. Commun. 2013, 49, 9407-9409. doi:10.1039/C3CC44452J

6. Noujeim, N.; Samsam, S.; Eberlin, L.; Sanon, S. H.; Rochefort, D.; Schmitzer, A. R. Soft Matter 2012, 8, 10914-10920. doi:10.1039/C2SM26213D

7. Bara, J. E.; Hatakeyama, E. S.; Wiesenauer, B. R.; Zeng, X.; Noble, R. D.; Gin, D. L. Liq. Cryst. 2010, 37, 1587-1599. doi:10.1080/02678292.2010.521859

8. Kumar, S.; Gupta, S. K. Tetrahedron Lett. 2010, 51, 5459-5462. doi:10.1016/j.tetlet.2010.08.022

9. D'Anna, F.; Noto, R. Eur. J. Org. Chem. 2014, 20, 4201-4223. doi:10.1002/ejoc.201301871

10. Pană, A.; Iliş, M.; Staicu, T.; Pasuk, I.; Cîrcu, V. Liq. Cryst. 2016, 43, 381-392. doi:10.1080/02678292.2015.1116630

11. Anderson, J. L.; Ding, R. F.; Ellern, A.; Armstrong, D. W. J. Am. Chem. Soc. 2005, 127, 593-604. doi:10.1021/ja046521u

12. Pană, A.; Iliş, M.; Micutz, M.; Dumitraşcu, F.; Pasuk, I.; Cîrcu, V. RSC Adv. 2014, 4, 59491-59497. doi:10.1039/C4RA11023D

13. Bhadani, A.; Misono, T.; Singh, S.; Sakai, K.; Sakai, H.; Abe, M. Adv. Colloid Interface Sci. 2016, 231, 36-58. doi:10.1016/j.cis.2016.03.005

14. Pietralik, Z.; Kołodziejska, Ż.; Weiss, M.; Kozak, M. PLoS One 2015, 10, e0144373. doi:10.1371/journal.pone.0144373

15. Zhou, T.; Llizo, A.; Li, P.; Wang, C.; Guo, Y.; Ao, M.; Bai, L.; Wang, C.; Yang, Y.; Xu, G. J. Phys. Chem. C 2013, 117, 26573-26581. doi:10.1021/jp4061363

16. Andrzejewska, W.; Wilkowska, M.; Chrabaszczewska, M.; Kozak, M. RSC Adv. 2017, 7, 26006-26018. doi:10.1039/C6RA24978G

17. Kamboj, R.; Singh, S.; Bhadani, A.; Kataria, H.; Kaur, G. Langmuir 2012, 28, 11969-11978. doi:10.1021/la300920p

18. Bhadani, A.; Singh, S. Langmuir 2011, 27, 14033-14044. doi:10.1021/la202201r
19. Rodrigues, M.; Calpena, A. C.; Amabilino, D. B.;

Garduño-Ramíreze, M. L.; Pérez-García, L. J. Mater. Chem. B 2014, 2 , 5419-5429. doi:10.1039/C4TB00450G

20. Ilincă, T. A.; Pasuk, I.; Cîrcu, V. New J. Chem. 2017, 41, 11113-11124. doi:10.1039/C7NJ02561K

21. Saielli, G.; Bagno, A.; Wang, Y. J. Phys. Chem. B 2015, 119, 3829-3836. doi:10.1021/jp5104565

22. Bruce, D. W.; Estdale, S.; Guillon, D.; Heinrich, B. Liq. Cryst. 1995, 19, 301-305. doi:10.1080/02678299508031983

23. Cruz, C.; Heinrich, B.; Ribeiro, A. C.; Bruce, D. W.; Guillon, D. Liq. Cryst. 2000, 27, 1625-1631. doi:10.1080/026782900750037185

24. Lava, K.; Evrard, Y.; Van Hecke, K.; Van Meervelt, L.; Binnemans, K. RSC Adv. 2012, 2, 8061-8070. doi:10.1039/C2RA21208K

25. Cardinaels, T.; Lava, K.; Goossens, K.; Eliseeva, S. V.; Binnemans, K. Langmuir 2011, 27, 2036-2043. doi:10.1021/la1047276

26. Haristoy, D.; Tsiourvas, D. Liq. Cryst. 2004, 31, 697-703. doi:10.1080/02678290410001675110

27. Mihelj, T.; Popović, J.; Skoko, Ž.; Tomašić, V. Thermochim. Acta 2014, 591, 119-129. doi:10.1016/j.tca.2014.07.024

28. Nikokavoura, A.; Tsiourvas, D.; Arkas, M.; Sideratou, Z.; Paleos, C. M. Liq. Cryst. 2002, 29, 1547-1553. doi:10.1080/0267829021000034808

29. Rettenmeier, E.; Tokarev, A.; Blanc, C.; Dieudonné, P.; Guari, Y.; Hesemann, P. J. Colloid Interface Sci. 2011, 356, 639-646. doi:10.1016/j.jcis.2011.01.055

30. Wang, Y.; Marques, E. F. J. Phys. Chem. B 2006, 110, 1151-1157. doi:10.1021/jp0552729

31. Wang, Y.; Marques, E. F. J. Therm. Anal. Calorim. 2010, 100, 501-508. doi:10.1007/s10973-009-0653-8

32. Westphal, E.; Henrique da Silva, D.; Molin, F.; Gallardo, H. RSC Adv. 2013, 3, 6442-6454. doi:10.1039/C3RA23456H

33. Lagerwall, J. P. F.; Scalia, G. J. Mater. Chem. 2008, 18, 2890-2898. doi:10.1039/b802707b

34. Rahman, M.; Lee, W. J. Phys. D: Appl. Phys. 2009, 42, 063001. doi:10.1088/0022-3727/42/6/063001

35. Dolgov, L.; Kovalchuk, O.; Lebovka, N.; Tomylko, S.; Yaroshchuk, O. Liquid crystal dispersions of carbon nanotubes: dielectric, electro-optical and structural peculiarities. In Carbon Nanotubes; Marulanda, J. M., Ed.; InTech: Rijeka, Croatia, 2010; pp 451-484. doi:10.5772/39439

36. Dierking, I.; Scalia, G.; Morales, P.; LeClere, D. Adv. Mater. 2004, 16, 865-869. doi:10.1002/adma.200306196

37. Rosu, C.; Manaila-Maximean, D.; Donescu, D.; Frunza, S.; Sterian, A. R. Mod. Phys. Lett. B 2010, 24, 65-73. doi:10.1142/S0217984910022238

38. Ganea, C. P.; Manaila-Maximean, D. UPB Sci. Bull., Ser. A 2011, 73, 209-216.

39. Blinov, L. M.; Chigrinov, V. G. Electrooptic Effects in Liquid Crystal Material; Springer: New York, NY, U.S.A., 1996. doi:10.1007/978-1-4612-2692-5

40. Koval'chuk, A. V.; Dolgov, L.; Yaroshchuk, O. Semicond. Phys., Quantum Electron. Optoelectron. 2008, 11, 337-341.

41. Chen, H.-Y.; Lee, W. Appl. Phys. Lett. 2006, 88, 222105. doi:10.1063/1.2208373

42. Lee, W.; Chen, H.-Y.; Shih, Y.-C. J. Soc. Inf. Disp. 2008, 16, 733-741.

43. García-García, A.; Vergaz, R.; Algorri, J. F.; Quintana, X.; Otón, J. M. Beilstein J. Nanotechnol. 2015, 6, 396-403. doi:10.3762/bjnano.6.39

44. Tomylko, S.; Yaroshchuk, O.; Koval'chuk, O.; Lebovka, N. Phys. Chem. Chem. Phys. 2017, 19, 16456-16463. doi:10.1039/C7CP01722G 
45. Lee, J. J.; Yamaguchi, A.; Alam, Md. A.; Yamamoto, Y.; Fukushima, T.; Kato, K.; Takata, M.; Fujita, N.; Aida, T. Angew. Chem., Int. Ed. 2012, 51, 8490-8494. doi:10.1002/anie.201203284

46. Hunt, P. A.; Ashworth, C. R.; Matthews, R. P. Chem. Soc. Rev. 2015, 44, 1257-1288. doi:10.1039/C4CS00278D

47. Dong, K.; Zhang, S.; Wang, J. Chem. Commun. 2016, 52, 6744-6764. doi:10.1039/C5CC10120D

48. Men, S.; Mitchell, D. S.; Lovelock, K. R. J.; Licence, P. ChemPhysChem 2015, 16, 2211-2218. doi:10.1002/cphc.201500227

49. Wulf, A.; Fumino, K.; Michalik, D.; Ludwig, R. ChemPhysChem 2007, 8, 2265-2269. doi:10.1002/cphc.200700508

50. He, H.; Chen, H.; Zheng, Y.; Zhang, S.; Yu, Z. Chem. Eng. Sci. 2015, 121, 169-179. doi:10.1016/j.ces.2014.07.024

51. Headley, A. D.; Jackson, N. M. J. Phys. Org. Chem. 2002, 15, 52-55. doi:10.1002/poc.449

52. Kremer, F.; Schönhals, A., Eds. Broadband Dielectric Spectroscopy; Springer-Verlag: Berlin-Heidelberg, Germany, 2003. doi:10.1007/978-3-642-56120-7

53. Dyre, J. C.; Maass, P.; Roling, B.; Sidebottom, D. L. Rep. Prog. Phys. 2009, 72, 046501. doi:10.1088/0034-4885/72/4/046501

54. Jacobs, J. D.; Koerner, H.; Heinz, H.; Farmer, B. L.; Mirau, P.; Garrett, P. H.; Vaia, R. A. J. Phys. Chem. B 2006, 110, 20143-20157. doi:10.1021/jp061931।

55. Klein, R. J.; Zhang, S.; Dou, S.; Jones, B. H.; Colby, R. H.; Runta, J. J. Chem. Phys. 2006, 124, 144903. doi:10.1063/1.2186638

56. Kumar, J.; Manjuladevi, V.; Gupta, R. K.; Kumar, S. Liq. Cryst. 2016, 43, 488-496. doi:10.1080/02678292.2015.1119320

57. García-García, A.; Vergaz, R.; Algorri, J. F.; Zito, G.; Cacace, T.; Marino, A.; Otón, J. M.; Geday, M. A. Beilstein J. Nanotechnol. 2016, 7, 825-833. doi:10.3762/bjnano.7.74

58. Jagadish, K.; Srikantaswamy, S.; Byrappa, K.; Shruthi, L.; Abhilash, M. R. J. Nanomater. 2015, 2015, No. 381275. doi:10.1155/2015/381275

\section{License and Terms}

This is an Open Access article under the terms of the Creative Commons Attribution License (http://creativecommons.org/licenses/by/4.0), which permits unrestricted use, distribution, and reproduction in any medium, provided the original work is properly cited.

The license is subject to the Beilstein Journal of Nanotechnology terms and conditions: (http://www.beilstein-journals.org/bjnano)

The definitive version of this article is the electronic one which can be found at: $\underline{\text { doi:10.3762/bjnano.9.19 }}$ 